\title{
Écrire, chanter, agir : les graduels et missels notés en notation aquitaine avant 1100
}

Thèse de doctorat sous la direction de M. Jean-Claude scHMITT, EHESS, Paris. Soutenue le 12 décembre 2011

\section{Eduardo Henrik Aubert}

\section{(2) OpenEdition}

12 Journals

Édition électronique

URL : https://journals.openedition.org/cem/12391

DOI : 10.4000/cem.12391

ISSN : 1954-3093

Éditeur

Centre d'études médiévales Saint-Germain d'Auxerre

Référence électronique

Eduardo Henrik Aubert, «Écrire, chanter, agir : les graduels et missels notés en notation aquitaine avant 1100 », Bulletin du centre d'études médiévales d'Auxerre | BUCEMA [En ligne], 16 | 2012, mis en ligne le 30 novembre 2012, consulté le 22 septembre 2022. URL : http://journals.openedition.org/cem/ 12391 ; DOI : https://doi.org/10.4000/cem.12391

Ce document a été généré automatiquement le 22 septembre 2022.

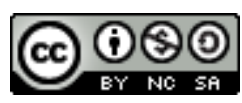

Creative Commons - Attribution - Pas d'Utilisation Commerciale - Partage dans les Mêmes Conditions 4.0 International - CC BY-NC-SA 4.0

https://creativecommons.org/licenses/by-nc-sa/4.0/ 


\section{Écrire, chanter, agir : les graduels et missels notés en notation aquitaine avant 1100}

Thèse de doctorat sous la direction de M. Jean-Claude ScHMITT, EHESS, Paris. Soutenue le 12 décembre 2011

\section{Eduardo Henrik Aubert}

1 La thèse étudie les graduels, graduels composites (graduels-lectionnaires et graduels-sacramentaires) et missels ayant été produits entre la fin du $\mathrm{IX}^{\mathrm{e}}$ et la fin du $\mathrm{XI}^{\mathrm{e}}$ siècle et notés en une notation, qui, depuis le XIX ${ }^{e}$ siècle, est appelée aquitaine. Son centre d'intérêt est la transformation de tels livres notés entre la période

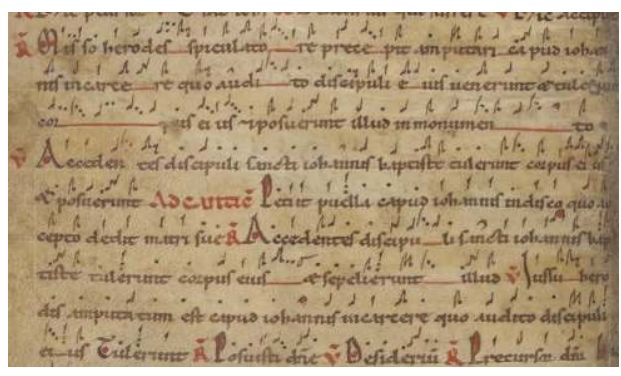
carolingienne tardive et les débuts de la réforme grégorienne. La proposition soutenue est que le changement qui conduisit de la transmission exclusivement orale à l'usage disséminé des livres de chant notés ne fut pas un développement limité à un prétendu domaine indépendant de la "musique ", moins encore à une progression inertielle de l'écriture, mais un élément et un agent dans les profonds changements vécus pendant cette période par la société de l'Occident médiéval. C'est pourquoi le titre enchaîne trois actions : on part de celle qui est le plus directement liée à l'évidence matérielle (écrire) ; on passe au domaine des pratiques les plus proches de ces évidences - ici évoquées par « chanter ", mais on se souviendra que, même aux niveaux les plus proches, ces pratiques «musicales » ne sont pas restreintes au seul phénomène de la production vocale - ; on arrive au domaine de la généralité du faire (agir), qui, dans la pluralité de ses actes, organisés en système d'action (ou agence), est responsable de la structuration de la société.

2 Si l'isolement de « l'écrire » a donné en large partie le sujet de la paléographie musicale traditionnelle - au moins depuis le XVII ${ }^{\mathrm{e}}$ siècle - et l'articulation entre écrire et chanter 
a fourni la matière de base à la musicologie historique classique - à partir du XIX ${ }^{e}$ siècle -, l'enchaînement de l'écrire au chanter et à l'agir ne s'annonce partiellement que vers les années 1970 et 1980 dans quelques travaux sur les notations neumatiques. Cette thèse essaie de faire avancer cette problématique en proposant de comprendre l'écriture de la musique comme étant une manière de faire des choses au moyen de l'écriture, l'un des multiples actes, qui, compris comme parties dialectiques d'un ensemble articulé, sont responsables pour donner forme et texture à la société, autrement dit, pour la structurer. Selon cette perspective, l'écriture musicale est moins un langage contenu en lui-même qu'un médium, "an extension of men" selon la définition de Marshall McLuhan (1911-1980). On arrive ainsi à la notion du signemédium, dont le postulat essentiel est que la vie sociale est construite par l'emploi de médias qui sont des types de " choses » qui tiennent le rôle de médiateurs d'un rapport social. Cette notion n'est pas opposée à celles qui correspondent à l'isolement heuristique de l'écriture - pour laquelle nous parlons de signe-représentation - ou à l'articulation entre écrire et chanter - que nous nommons «signe-trace»: elle s'intéresse à les dépasser de l'intérieur et par accumulation. C'est ainsi que j'ai conçu ma thèse en six chapitres, dont les trois premiers sont pensés comme un préambule nécessaire aux trois derniers.

3 Le premier chapitre est un catalogue raisonné des graduels, graduels composites et missels notés en notation aquitaine avant 1100. Son centre d'intérêt est fondamentalement paléographique et il s'attache, ainsi, plus directement, à la question de l'écrire, sans accumuler, dans un premier moment, les problèmes soulevés par la progression vers le chanter et l'agir. Le chapitre fournit les bases sans lesquelles il serait impossible de continuer l'argument: vue d'ensemble de la documentation existante, dénombrement des scribes et organisation de leur travail, datation des sources. J'ai essayé de regrouper ici tous les documents, livres complets et fragments, qui rentraient dans la triple délimitation chronologique (avant 1100), du type de livre (graduels et missels) et du type de notation (aquitaine). Ceci a donné un corpus de 26 témoins, dont 5 livres complets et fragments de 21 livres, de taille fort inégale. Je conçois, dans tous les cas, le catalogue comme un corpus ouvert, car même en ayant regroupé tous les témoins précédemment connus et ceux que j'ai pu découvrir, une exploration systématique de toutes les bibliothèques et, notamment, de tous les fonds d'archives, est susceptible d'élargir notre connaissance de documents de ce genre.

Dans les deux chapitres suivants, j'approche le passage de l'écrire au chanter. Le deuxième chapitre est tout entier consacré à une exploration de la sémiologie de la notation aquitaine "classique » $\mathrm{du} \mathrm{XI}^{\mathrm{e}}$ siècle, la sémiologie étant conçue ici comme recherche de la "raison (logos) de la diversité des signes (semeion)» (Dom Eugène Cardine, 1905-1988) dans la représentation d'un « même » contenu mélodique. Avec ce travail, je cherche à déterminer non seulement les différentes modalités de codage du référent musical dans les manuscrits aquitains, mais aussi à appréhender comment la différence dans l'écriture, dans un même manuscrit ou entre des manuscrits différents, permet de concevoir le travail de l'écriture en rapport au fait musical : copie pure et simple d'un exemplaire ou recodage dans le présent motivé par le fait musical - et, question qui ne s'annonce que comme une conclusion logique, par la médiation du scribe ? Tous les manuscrits examinés, exception faite du manuscrit FIRENZE, BibliotecaMedicea-Laurenziana, Ashburnham 62, même les plus tardifs, démontrent un souci pour représenter, dans les limites de chaque système graphique, des éléments de 
l'organisation rythmique de la mélodie. En même temps, ces stratégies, même dans le cas des manuscrits rapprochés entre eux, sont diverses. Ceci amène au constat que, dans la notation de ces graduels, les différents scribes ont, indépendamment de la simple copie d'un modèle d'agencement rythmique des neumes, été sensibles dans leur pratique d'écriture à la perception du phénomène musical et ont cherché à trouver, de manière souvent originale, des solutions pour mettre cette perception par écrit. Le scénario est donc, de manière générale, celui d'un engagement actif avec la notation, jusqu'à la fin du XI $\mathrm{I}^{\mathrm{e}}$ siècle, au moins, en tout cas pour toute la période envisagée dans la thèse.

5 Le troisième chapitre prend son point de départ du constat déjà fait au cours du chapitre précédent, celui de l'existence de différences non seulement au niveau du codage, mais dans le fait musical même, autant qu'on peut l'appréhender au moyen de l'écriture. Il pose ainsi le problème des variantes mélodiques dans les graduels aquitains. Cette étude contribue à mieux classer les graduels aquitains et à comprendre leurs rapports les uns aux autres, et les rapports aux différentes traditions régionales du chant grégorien. Elle permet aussi d'approcher le terrain délicat des rapports entre différence écrite et différence chantée, un rapport qui ne va pas de soi et qui empêche toute lecture simpliste du signe comme reflet transitif du référent. La méthodologie employée ici est l'héritière de celle avancée dans le travail pionnier des moines de Solesmes, mais elle est changée à la fois pour s'adapter au problème spécifique des graduels aquitains et pour fonder les résultats sur une grille plus précise. Un des principaux soucis a été celui de ne pas confondre toutes les variantes comme si elles avaient le même statut. Il m'a semblé possible de distinguer entre des variantes, qui s'expliquent en fonction de la conformité ou non-conformité par rapport à une variable - et dont toute la problématique peut se résumer dans une formulation qui sépare «oui » et «non" - et des variantes dans lesquelles, s'il y a "variable», elles sont multiples, car les possibilités de variation sont (au moins potentiellement) beaucoup plus vastes que la simple alternative fournie par le couple oui/non. J'ai proposé d'appeler les dernières "variantes de base", et les premières "variantes procédurales ». Parmi les variantes procédurales, j'ai identifié trois types: celles dues au phénomène de l'initio debilis, les remplissages de tierce et le choix entre $\mathrm{E} / \mathrm{b}$ et $\mathrm{F} / \mathrm{c}$ dans certains contextes précis.

6 C'est avec les trois derniers chapitres que j'aborde l'ensemble du spectre écrire/ chanter/agir. Devant des problèmes concrets posés par l'écriture de trois livres, ces trois études de cas proposent une description détaillée qui essaie de dégager l'argument que ces manuscrits sont des faits matériels inextricablement liés à la médiation du fait musical dans l'écriture par le scribe. Ils évoquent donc le paradigme du signe-médium et la question de l'agir (au singulier) ou de l'agence (comme système structuré d'action) du scribe. Les livres choisis pour cette étude ont été produits à différentes époques dans le spectre temporel considéré par cette thèse. Chaque chapitre introduit en quelque sorte au problème de la conception de l'écriture musicale en tant qu'agir, ceci par deux procédés fondamentaux: l'examen détaillé du fait graphique en question et l'indication, aussi précise que possible, du contexte concret de production de chacun des livres.

7 Le quatrième chapitre traite du manuscrit ALBI, Bibliothèque municipale, ms. 44, un graduel-antiphonaire de la fin $\mathrm{du} \mathrm{IX}^{\mathrm{e}}$ ou du début du $\mathrm{x}^{\mathrm{e}}$ siècle. La question posée par ce manuscrit est celle d'une notation éparse (peu de pièces notées) et souvent partielle - 
notation de segments à l'intérieur d'une pièce. Se confrontant au problème central du fonctionnalisme - à quelle fin ? pour quelle fonction? - le chapitre essaie de partir du dedans de la pratique des scribes pour soutenir que la fonction n'est pas simplement un préalable à l'écriture, mais aussi son produit, et donc que l'agir est producteur de système. Je propose de concevoir le travail de notation en trois strates distinctes et, notamment, de voir la transformation entre les strates et à l'intérieur de chacune d'entre elles comme un fait essentiel à la compréhension de ce qui fait et de ce qu'est la notation neumatique. Je relève, en particulier, l'emploi créatif et sans cesse renouvelé des neumes dans la première strate, par le scribe principal, comme caractéristique d'un rapport créatif et dialectique entre chanter et écrire, où l'écriture musicale sert souvent de support ouvert de création musicale, de plateforme créative, pourrait-on dire. Je propose, en conclusion, que cette manière d'utiliser l'écriture neumatique se situe dans un ensemble plus large, celui des pratiques de l'écriture qui font structure en tant que système d'action (ou agence) avec un ensemble d'autres pratiques. À partir de l'identification de la main du même scribe dans un autre manuscrit, je propose des arguments pour soutenir l'hypothèse que ce manuscrit a été copié dans la cathédrale d'Albi vers la fin du IX siècle ou, tout au plus, au début du $\mathrm{x}^{\mathrm{e}}$ siècle.

8 Le cinquième chapitre traite du manuscrit FIRENZE, Biblioteca Medicea-Laurenziana, Ashburnham 62, un fragment de graduel, comptant 50 feuillets, daté du deuxième tiers $\mathrm{du} \mathrm{XI} \mathrm{I}^{\mathrm{e}}$ siècle. Il soulève un problème capital, car il semble faire un mauvais usage de l'un des instruments les plus importants de la notation aquitaine du $\mathrm{XI}^{\mathrm{e}}$ siècle, l'emploi d'une ligne sèche pour donner un sens mélodique stable à la position verticale des signes dans la longueur de (au moins) une ligne d'écriture. Au moyen d'une description de tous les chants notés intégralement dans le manuscrit, et, en lien avec une autre particularité de l'écriture, un signe qui indique le degré inférieur dans l'intervalle d'un demi-ton, je propose de voir ici la trace d'une appréhension hétérogène du fait musical par le scribe, qui présuppose une compréhension de celui-ci ancrée dans l'expérience. À partir de cette étude, en concevant ces faits graphiques comme une sorte d'« analyse musicale indicielle » ou " pragmatique ", j'avance une définition pour quatre notions : phrase, motif, thème, "formulisme", qui me semblent opératoires en matière d'analyse du chant grégorien et qui ont l'avantage de découler de la pratique du scribe. Je propose ensuite de dégager trois modes graphiques dans le manuscrit, différenciés selon le répertoire : le systématisme explicite, le systématisme implicite, l'épisodisme. Enfin, je propose de localiser ce manuscrit dans la mouvance des réformes monastiques $\mathrm{du} \mathrm{XI} \mathrm{e}^{\mathrm{e}}$ siècle et, plus précisément, à Saint-Martin de Limoges, dans le deuxième tiers du $\mathrm{XI}^{\mathrm{e}}$ siècle.

9 Le sixième chapitre traite du manuscrit LoNDON, British Library, Harley 4951, un gradueltonaire du dernier tiers $d u \mathrm{XI}^{\mathrm{e}}$ siècle. L'analyse se fait en deux parties : la première traite des nombreuses corrections affleurant dans l'écriture neumatique, et la seconde des quinze notations en marge. En ce qui concerne la première partie, dans quelques cas seulement, la correction a pu être regardée comme une erreur de fait, au sens où il n'y avait aucune « intentionnalité » ou " propos » dans la version effacée. Plus souvent, nous sommes devant deux versions d'un passage spécifique et le scribe est en fait en train de choisir parmi des possibilités - sinon de les créer. Il est donc en train de se poser en tant qu'intermédiaire (filtre sélectif) de traditions diverses et de créateur qui " médialise » la tradition qu'il crée lui-même dans le médium de l'écrit. C'est donc au sens concret de médiation entre des sphères distinctes que nous devons comprendre 
qu'il n'y a pas un seul niveau de variance ou d'invariance, mais plutôt des strates multiples dans lesquelles la variation peut être placée. D'autre part, dans la seconde partie du chapitre, j'examine le groupe fort intéressant des quinze notations entrées dans les marges par le scribe principal et par un autre scribe. Si le travail du scribe principal en marge est, pour la plupart, une continuation de ce qu'il a fait au moyen d'effacements et de réécritures, les interventions marginales du correcteur proposent en général la version partagée par l'ensemble des autres sources aquitaines contre une version singulière du scribe principal. Le fait qu'il s'agit d'une autre personne et que le scribe principal n'a pas pensé à expliciter la version partagée dans la marge permet de caractériser ces interventions comme conflictuelles, au moins au sens où le correcteur indique ainsi que la révision du répertoire - en général ou pour ces cas spécifiques - ne va pas de soi. L'analyse de toutes les corrections et de toutes les entrées en marge du manuscrit nous amène à proposer un cadre plus nuancé pour l'étude concrète des variantes, axé sur les notions d' " actes de parole ", «norme » et "système ", à partir des travaux du linguiste roumain Eugen Coseriu (1921-2002), qui nous semble plus adapté à concevoir le travail de production et de transmission des variantes comme une forme de la communication sociale. En même temps, j'avance une interprétation historique particulière pour l'origine de cette source : écrite à Moissac pour SaintSernin de Toulouse, passée ensuite à Saint-Étienne de Toulouse.

Dans la conclusion de ma thèse, je remets en perspective historique les trois études de cas qui ont fourni le cœur du travail, dans les chapitres 4,5 et 6 . Il s'agit, d'une part, de caractériser les différentes formes de l'agence - cas d'espèce de la morphologie historique de l'agir - qui se déduisent de l'analyse des trois sources. Ainsi, il s'agit de caractériser le passage d'un état donné dans Albi 44, où l'écriture est une plateforme créative par où passe l'agence, où l'agentivité - la possibilité de faire agence - peut se communiquer sans que l'écrit devienne dépositaire d'une agence, à l'état trouvé dans Harley 4951, où l'action se réalise par et (en grande partie) dans l'écriture, quand l'écriture musicale devient un élément fondamental de l'opposition entre personne et tradition collective. Cette transformation s'est faite en passant par Ashburnham 62, où l'écrit est beaucoup plus présent que dans Albi 44. Sans en faire une téléologie, la diffusion de l'écriture musicale, au cours du deuxième tiers du xI siècle, a certainement joué un rôle dans le processus qui a postérieurement amené à en faire un lieu fort de transmission de l'agence. Il fallait qu'elle fût présente et ouverte à la manipulation; il fallait qu'elle fût aussi disponible.

11 La conclusion traite, dès lors, d'un essai de "contexturalisation", un mot que j'ai proposé pour le distinguer de "contextualisation", qui présuppose souvent une distinction ontologique entre le particulier et le général; entre ces deux registres discursifs, je ne vois que la progression interprétative par laquelle on peut «tisser ", sur le plan heuristique, des rapports entre des éléments contigus sur le même plan de réalité. C'est ainsi que je pars des sources mêmes et que je vois dans des éléments matériellement proches la possibilité d'ouvrir la considération de la morphologie de l'agence vers d'« autres" domaines. Comme souvent les manuscrits eux-mêmes (ou leurs scribes) ne sont pas purement musicaux, mais contiennent des éléments d'autres champs de l'expérience, j'ai rapproché la morphologie de l'action de l'écriture musicale de celles de l'homélie et du droit canon dans le cas d'Albi 44; des coutumiers monastiques dans le cas d'Ashburnham 62; et des cartulaires dans le cas de Harley 4951. En même temps, j'ai suggéré que, dans mon corpus même, chaque source explorée dans les chapitres 4,5 et 6 trouvait des parallèles et des points d'ancrage dans des sources 
chronologiquement proches. J'estime que le prolongement conséquent de ces " contexturalisations ", à la fois dans les sources musicales et dans celles plutôt liées à d'autres champs d'activité, serait la manière la plus profitable pour faire avancer la problématique de la recherche.

Reçu : 4 septembre 2012 - Accepté : 5 septembre 2012

\section{AUTEUR}

\section{EDUARDO HENRIK AUBERT}

University of Cambridge, Faculty of Music 